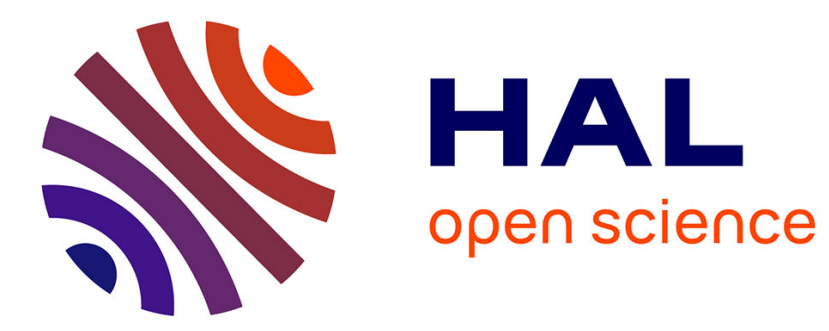

\title{
Les Fours céramiques en Gaule romaine. Étude morphologique \\ Pascal Duhamel
}

\section{To cite this version:}

Pascal Duhamel. Les Fours céramiques en Gaule romaine. Étude morphologique. École pratique des hautes études. 4e section, Sciences historiques et philologiques, 1974, 106 (1), pp.833-836. 10.3406/ephe.1974.5957 . hal-01851115

\section{HAL Id: hal-01851115 https://hal.science/hal-01851115}

Submitted on 29 Jul 2018

HAL is a multi-disciplinary open access archive for the deposit and dissemination of scientific research documents, whether they are published or not. The documents may come from teaching and research institutions in France or abroad, or from public or private research centers.
L'archive ouverte pluridisciplinaire HAL, est destinée au dépôt et à la diffusion de documents scientifiques de niveau recherche, publiés ou non, émanant des établissements d'enseignement et de recherche français ou étrangers, des laboratoires publics ou privés. 


\section{Les Fours céramiques en Gaule romaine. Étude morphologique} Pascal Duhamel

\section{Citer ce document / Cite this document :}

Duhamel Pascal. Les Fours céramiques en Gaule romaine. Étude morphologique. In: École pratique des hautes études. 4e section, Sciences historiques et philologiques. Annuaire 1973-1974. 1974. pp. 833-836;

doi : https://doi.org/10.3406/ephe.1974.5957

https://www.persee.fr/doc/ephe_0000-0001_1973_num_1_1_5957

Fichier pdf généré le 17/05/2018 


\title{
LES FOURS CERAMIQUES EN GAULE ROMAINE ETUDE MORPHOLOGIQUE (1)
}

\author{
par Pascal Duhamel \\ Directeur d'études : M. Paul-Marie Duval
}

Depuis plus d'un siècle, les fouilles archéologiques ont mis au jour près de $\mathbf{2 . 0 0 0}$ fours de cuisson céramique gallo-romains. Cependant, extrêmement fréquents, ces vestiges restaient méconnus malgré l'abondance d'une littérature d'ailleurs inégale et fort dispersée. Il devenait nécessaire d'en rassembler les documents et de tenter un étude systématique permettant d'éclairer nos connaissances des produits céramiques comme du travail de manufacture.

Le présent ouvrage, accompagné de notes et figures et suivi d'une bibliographie générale, est divisé en cinq chapitres; il est complété par un inventaire géographique des ateliers céramiques recensés.

I. Présentation : un bilan des connaissances antérieures fait apparaître le nombre des carences et leurs incidences dans le renouvellement de conceptions erronées. Ainsi se dessinent les principaux objectifs de l'étude, qui, d'une connaissance plus exacte des fours, débouche sur une meilleure approche des problèmes économiques et sur certains aspects de la diffusion des techniques. L'ampleur des résultats acquis a d'ailleurs rendu nécessaire la limitation du sujet à la seule morphologie des fours.

II. Méthodologie : en premier lieu sont exposées les difficultés d'une telle analyse. Si les différentes sources (archéologiques, historiques, ethnographiques et renseignements techniques) restent complémentaires, les vestiges archéologiques constituent la source principale malgré la fréquente imprécision

(1) Cette thèse, déposée le 8 avril 1973, par M. Pauł-Marie Duval, directeur d'études, a valu à M. Pascal Duhamel, par délibération de l'Assemblée de la Section en date du 17 juin 1973, rendue sur le rapport de MM. Raymond Bloch et Venceslas Kruta, le titre d'élève diplómé de la IVe Section de l'École pratique des Hautes Etudes. 
des comptes rendus des fouilleurs. Une revue des sources historiques montre l'intérêt de ces documents (et surtout des figurations), malheureusement bien rares. L'utilisation des autres sources reste assujettie à d'extrêmes précautions analytiques.

Le stockage et le traitement ont nécessité des méthodes appropriées à la nature de ces informations. Parmi celles-ci, on notera des systèmes d'intégration des données de type structural, de fréquentes analyses statistiques, des méthodes de sériation graphique et l'étude des variations concomitantes. Les interprétations finales restant soumises à certaines conditions de validité, notamment pour la chronologie, certains des résultats peuvent n'être que présomptions.

III. Technologie : indispensables à une bonne compréhension des fours céramiques, quelques éléments technologiques sont fournis dans ce chapitre : nature et propriétés des argiles, principe de la combustion, phénomènes cristallins et oxydoréducteurs de la cuisson. Cela permet enfin d'envisager le principe de la cuisson dans un four céramique.

IV. Analyse des fours : constituant le corps de l'étude, ce chapitre est présenté en sept parties dont on ne peut donner ici qu'un aperçu :

10 évolution des procédés de cuisson : un bref schéma vise à réinsérer les installations gallo-romaines dans le cadre de la progression des connaissances techniques et des structures socio-économiques;

$2^{\circ}$ les découvertes de fours en Gaule romaine : très nombreuses (plus de 1.500 sur près de 400 ateliers), elles consistent en vestiges souvent incomplets. Ces méthodes de détection et d'interprétation s'affirment, tandis que le nombre des découvertes devra croître encore puisqu'une estimation ici tentée porte à un minimum de $\mathbf{1 0 . 0 0 0}$ le nombre des fours utilisés;

30 principe du four gallo-romain : le fonctionnement du four-type gallo-romain est ensuite défini avec le rôle de chacune des parties et la terminologie employéc;

4. 0 morphologie : éléments pour une classification; trois aspects fondamentaux ont été utilisés et constituent la base d'une classification qui reste ouverte à d'autres types de fours : la catégorie fonctionnelle, la forme et le dispositif de la chambre inférieure. 
L'étude de la forme, particulièrement fructueuse, remet en cause de nombreuses conceptions erronées. On notera particulièrement que, plus fréquents qu'on ne le croit, les fours à sole rectangulaire sont, pour $72 \%$, des fours à poterie et qu'ils sont majoritaires dans les ateliers de sigillée. Leur diffusion chronogéographique atteste leur corrélation avec la progression de techniques italiques importées (développement en milieu fortement romanisé, utilisation pour la fabrication de produits de tradition non gauloise, etc.).

L'examen du dispositif de la chambre inférieure est essentiel; le système adopté traduit une plus ou moins grande adaptation aux problèmes techniques (mécaniques et thermiques) et constitue un indice précis de l'évolution et de la transmission des techniques. Leurs différents types distingués paraissent en rapport avec la forme, les dimensions et la mise en œuvre du four. On remarquera spécialement la famille des fours à canal principal distribuant des canaux secondaires; ces dispositifs, utilisés tant sur des fours circulaires que des fours rectangulaires, sont sujets à une évolution chronologique et leur diffusion atteste une origine italique très probable, suivie d'un développement en milieu industriel dans les fabriques de terre sigillée principalement.

Ces analyses conduisent à la détermination de caractères propres à certaines productions céramiques et permettent d'envisager des phases de développement économique;

$5^{\circ}$ parties du four : chacun des éléments composant le four, après établissement d'une terminologie critique, est décrit; des exemples sont comparés et les problèmes d'interprétation envisagés. Au cours de cette revue surgissent des questions d'ordre technique : tirage, couverture des fours, problèmes de construction, etc.;

$6^{\circ}$ matériaux et mise en œuvre : les trois groupes de matériaux principaux envisagés (argile, tuiles-briques-carreaux, roches) traduisent une hiérarchie dans laquelle chacune des catégories inclut les matériaux de la précédente. Plus encore, les relations avec les formes, les productions et les dispositifs mettent en évidence la validité de cette hiérarchie pour l'évolution technique. Ainsi, dans les catégories successives, les fours munis de canaux sont représentés par les pourcentages de $0 \%, 50 \%, 55 \%$; les fours ayant cuit des terres sigillées par $0 \%, 34 \%, 61 \%$. Ces variations concomitantes nous fournissent des indices 
qui pourront peut-être ultérieurement servir à lever les incertitudes actuelles portant sur des installations incomplètes;

$7^{\circ}$ dimensions des fours : mesurées sur la sole, surface utile du four, elles ont été sériées par une répartition graphique. Les différents groupes de fours apparaissent nettement sous la forme de nébuleuses de disposition caractéristique par rapport à certains axes. Si ces nébuleuses caractérisent des groupes de fours de même forme, il a été aussi possible de sérier ces derniers ensembles et de faire apparaître une relation entre la largeur de la sole et le dispositif inférieur du four.

V. Conclusions. L'ensemble des résultats obtenus a permis de connaître de façon beaucoup plus précise les fours utilisés en Gaule romaine, mais constitue plus encore une somme d'éléments permettant d'étudier l'évolution technique et économique de l'industrie céramique. Les fours céramiques apparaissent, en outre, comme d'utiles témoins de la progression de techniques importées, indices indirects de la pénétration des influences romaines en Gaule. 